\title{
Prevalencia de infecciones de transmisión sexual en mujeres embarazadas atendidas en un hospital público de Chile
}

\author{
Alfredo Ovalle, María A. Martínez, Felipe de la Fuente, Nicolás Falcon, \\ Felipe Feliú, Felipe Fuentealba y Romina Gianini
}

\section{Prevalence of sexually transmitted infections in pregnant women attending a public hospital in Chile}

Introduction: The epidemiologic rates of gonorrhea have declined steadily in Chile, while the incidence of infections with Trichomonas vaginalis and Chlamydia trachomatis is not well known. Aim: Since these sexually transmitted infections (STIs) are associated with adverse pregnancy outcomes and perinatal infections, this study aimed to update their prevalence in a public hospital in the Metropolitan Region of Chile. Patients and Methods: Between April and October 2010 and April and October 2011, pregnant women attending the antenatal Service, Hospital San Borja Arriarán, were randomly selected for detection of T. vaginalis, N. gonorrhoeae and C. trachomatis by culture in modified Diamond's broth, Thayer-Martin agar, and by ompl gene amplification by nested PCR, respectively. We excluded pregnant women who received antibiotics within the past 30 days. Results: Two hundred and fifty five cervicovaginal samples were analyzed. C. trachomatis was detected in $15(5.9 \%)$ and $T$. vaginalis in $6(2.4 \%)$. N. gonorrhoeae was not found. Conclusion: The results show low prevalence of C. trachomatis and $T$. vaginalis and absence of $N$. gonorrhoeae. These rates have remained stable at this medical center since the 1990s, with a slight increase in C. trachomatis.

Key words: Sex transmitted infections, pregnancy, Chile.

Palabras clave: Infecciones de transmisión sexual, mujeres embarazadas, Chile.

\section{Introducción}

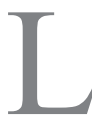

a Organización Mundial de la Salud OMS) estima que más de 340 millones de casos nuevos de infecciones de transmisión sexual (ITS) bacterianas y por Trichomonas vaginalis ocurren anualmente en el mundo ${ }^{1}$. Durante la gestación, las infecciones causadas por estos microorganismos han sido asociadas con aborto, parto prematuro, muerte fetal, bajo peso al nacer ${ }^{1-3}$ y pueden ser transmistidas verticalmente causando morbilidad y mortalidad neonatal ${ }^{1-3}$. Debido a que el diagnóstico de laboratorio de estos microorganismos, con la excepción de sífilis, requiere procedimientos de alto costo o laboratorios especializados, la elección de las estrategias de control de estos agentes debe incluir el conocimiento de su magnitud en la población local $^{4-5}$.

Chlamydia trachomatis es la bacteria de transmisión sexual más frecuente en el mundo, con una mayor prevalencia en adolescentes y adultos jóvenes de ambos géneros $^{1-4,6-8}$. Dado que la infección en la mujer es en hasta $80 \%$ de los casos asintomática, constituye un factor de riesgo acumulativo de enfermedad inflamatoria pélvica (EIP) y de secuelas reproductivas ${ }^{2-4,9}$.
Hasta hace unos años, se consideró incierto el papel de $C$. trachomatis sobre efectos adversos durante el embarazo $^{3}$. Actualmente, ha sido asociada con embarazo ectópico, aborto, parto prematuro y rotura prematura de membranas $^{2-3,10-12}$. El neonato adquiere la bacteria en su paso por el canal del parto y tiene riesgo de desarrollar conjuntivitis en $25-50 \%$ de los expuestos y hasta $17 \%$ de ellos desarrolla una neumonía ${ }^{13-15}$.

Neisseria gonorrhoeae causa infección cervical asintomática en aproximadamente $45 \%$ de los casos. $\mathrm{Si}$ no es detectada y tratada oportunamente puede producir graves secuelas ginecológicas y resultados adversos en el embarazo y en el neonato ${ }^{2-4,16}$. Hasta $35 \%$ de los embarazos en madres con infección gonocóccica no tratada resultan en aborto, parto prematuro y hasta $10 \%$ en muerte perinatal $^{2-4}$. En ausencia de profilaxis, los neonatos infectados al nacer pueden desarrollar conjuntivitis neonatal y ocasionalmente infección gonocóccica diseminada ${ }^{2}$. Comparativamente con otras regiones del mundo, las tasas de incidencia de infección gonocóccica en Chile son bajas, siendo por ello una causa infrecuente de infección femenina y de conjuntivitis neonatal ${ }^{8,17,18}$.

Trichomonas vaginalis es el patógeno de transmisión

\author{
Universidad de Chile, \\ Santiago, Chile. \\ Facultad de Medicina \\ Hospital San Borja Arriarán \\ Servicio y Departamento de \\ Obstetricia, Ginecología y \\ Neonatología (AO). \\ Instituto de Ciencias Biomédicas \\ Programa de Microbiología y \\ Micología (MAM, F de la F, \\ $N F, F F, F F, R G$. \\ Recibido: 12 de enero de 2012 \\ Aceptado: 6 de agosto de 2012 \\ Los autores declaran no tener \\ conflictos de interés con institución \\ alguna. \\ El estudio fue financiado \\ por recursos generados por sus \\ autores principales.


sexual no viral más frecuente en el mundo, con alrededor de 180 millones de casos nuevos anualmente, según estimaciones de la $\mathrm{OMS}^{6}$. Infecta principalmente el epitelio genital escamoso y una vez establecido puede persistir por largos períodos en la mujer ${ }^{19}$. Durante la gestación, la infección por este protozoo es un factor de riesgo de parto prematuro, niños de bajo peso al nacer y puede ser transmitida de manera vertical al recién nacido, pudiendo ocasionalmente causar enfermedad neonatal ${ }^{20,21}$ En la mujer la infección es asintomática en 25 a 50\% y los signos y síntomas clínicos varían ampliamente por lo que el diagnóstico de laboratorio es fundamental para su detección ${ }^{19}$.

El objetivo de este estudio fue actualizar la prevalencia de infecciones por $C$. trachomatis, $N$. gonorrhoeae y T. vaginalis en mujeres embarazadas atendidas en un Consultorio de Alto Riesgo Obstétrico.

\section{Pacientes y Métodos}

Población estudiada: Desde abril a octubre de 2010 y desde abril a octubre de 2011 se tomaron muestras cérvico-vaginales a 258 mujeres embarazadas atendidas en el Consultorio de Alto Riesgo Obstétrico (ARO), policlínica de infecciones del Hospital San Borja Arriarán. Esta unidad de infecciones atiende exclusivamente mujeres embarazadas con infecciones cérvico-vaginales o urinarias y pacientes con antecedente de resultados adversos (aborto, parto prematuro, mortalidad perinatal y morbilidad neonatal) asociados con infección bacteriana ascendente.

Las pacientes fueron seleccionadas al azar y no presentaron factores de riesgo ni características sospechosas para ITS. Por conveniencia fueron enroladas en forma consecutiva un día a la semana (5 pacientes por semana).

Criterios de inclusión: Mujeres embarazadas consultantes en ARO, con o sin signos o síntomas de infección genital.

Criterio de exclusión: Uso de antimicrobianos locales o sistémicos en los 30 días previos a la admisión.

El estudio fue aprobado por el Comité de Ética de la Facultad de Medicina de la Universidad de Chile y las pacientes dieron previamente su consentimiento informado.

Obtención y transporte de muestras clínicas: Se obtuvieron con especuloscopio dos muestras del flujo adyacente al cérvix y fondo de saco vaginal para diagnóstico de $C$. trachomatis, $N$. gonorrhoeae y $T$. vaginalis con tórulas estériles. La primera tórula fue inoculada en 1,5 ml de tampón sacarosa fosfato (2SP) para efectuar examen microscópico al fresco de T. vaginalis y extracción de ADN para diagnóstico de $C$. trachomatis por reacción de polimerasa en cadena (RPC). La tórula 2 fue inoculada en medio de transporte Amies (Oxoid) y luego utilizada en el cultivo de $N$. gonorrhoeae y de $T$. vaginalis. Las muestras fueron transportadas al laboratorio a temperatura ambiente dentro de las $2 \mathrm{~h}$ de obtenidas, e inmediatamente procesadas.

Diagnóstico microbiológico: Para el diagnóstico de $N$. gonorrhoeae, las muestras clínicas fueron inoculadas en agar Thayer-Martin e incubadas a $35,5{ }^{\circ} \mathrm{C}$ en una atmósfera húmeda con $3 \%$ de $\mathrm{CO}_{2}$ por $48 \mathrm{~h}$. Las placas de agar Thayer-Martin fueron preparadas cada quince días, efectuándose control microbiológico con una cepa de $N$. gonorrhoeae perteneciente al cepario del programa de Microbiología. Para el examen al fresco de T. vaginalis se depositó una gota del tampón 2SP en un portaobjeto, observándose la preparación al microscopio óptico con aumento de 400 X. Para el diagnóstico mediante cultivo, las muestras fueron inoculadas en caldo Diamond modificado e incubadas a $35,5^{\circ} \mathrm{C}$, en aerobiosis por 6-7 días. Alícuotas del medio fueron observadas al microscopio con 400 X cada 48 h en busca de trofozoitos móviles ${ }^{22}$. El diagnóstico de C. trachomatis fue efectuado por amplificación del ADN mediante RPC anidada, de acuerdo a procedimientos previamente descritos ${ }^{7,15}$. La extracción del ADN fue efectuada procesando $200 \mu$ l de la muestra en tampón 2SP mediante el sistema comercial Wizard, SVgenomic DNA purification system ${ }^{\mathrm{TM}}$ (Promega). Se incluyeron controles positivos (muestras positivas obtenidas de estudios anteriores) y negativos (agua) en cada corrida de RPC. Para descartar la presencia de inhibidores del proceso de amplificación, en cada muestra se amplificó $327 \mathrm{pb}$ del gen $\beta$-globina ${ }^{7,15}$. Los productos de amplificación fueron analizados por electroforesis en geles de agarosa al 1,5\% y la visualización se realizó en transiluminador de luz U.V. Para evitar contaminaciones en las reacciones de RPC se aplicaron medidas de prevención estándares ${ }^{7,15}$.

\section{Resultados}

Se analizaron 258 muestras cérvico-vaginales obtenidas consecutivamente. Tres muestras fueron excluidas por empleo de antimicrobianos en los últimos 30 días. La edad materna promedio fue de 27,7 años, con un rango entre 14 y 43 años. Ciento ochenta y siete $(73,3 \%)$ fueron multíparas y 68 (26,7\%) primíparas.

Se incluyeron 255 muestras. C. trachomatis fue detectada en 15/255 (5,9\%) gestantes. En la totalidad de las muestras negativas el control de amplificación del gen $\beta$-globina fue positivo. T. vaginalis fue diagnosticada en $6 / 255(2,4 \%)$ casos, en $3(50 \%)$ en el examen al fresco y en todas ellas por cultivo. $N$. gonorrhoeae no fue aislada de las muestras. 


\section{Discusión}

Los resultados de este estudio demuestran una baja prevalencia de C. trachomatis y de $T$. vaginalis y ausencia de $N$. gonorrhoeae en mujeres embarazadas atendidas en un consultorio de infecciones.

La inexistencia de $N$. gonorrhoeae en nuestra muestra no es de extrañar dada la baja tasa de incidencia de este microorganismo en nuestro país. En un estudio reciente efectuado mediante RPC y en el que se enroló mujeres adolescentes de distintos estratos socio económicos tampoco se detectó esta bacteria ${ }^{8}$. Sin embargo, la muestra de elección para el diagnóstico por cultivo de esta bacteria es la muestra de endocervical, por lo que no se pueden descartar resultados falsamente negativos ${ }^{4}$.

Con respecto a $C$. trachomatis, la literatura médica nacional informa que la frecuencia de infección cervical en población general de mujeres embarazadas es 0 a $2 \%$ en rotura prematura de membranas de pretérmino, $3,8 \%$ en rotura prematura de membranas de término y $4,8 \%$ en parto prematuro con membranas intactas ${ }^{3}$.

Para observar la tendencia epidemiológica ${ }^{23}$, los datos de este estudio fueron comparados con aquellos obtenidos en un trabajo anterior efectuado en el mismo centro asistencial (policlínica de infecciones) y que incluyó análisis de 156 muestras clínicas, 82 en mujeres embarazadas de riesgo con edades y paridad similares. Se encontró un leve aumento de $C$. trachomatis (1,2 vs 5,9\% actual), en tanto que la frecuencia de $T$. vaginalis permaneció invariable. La detección de C. trachomatis en la serie anterior se efectuó por aislamiento en cultivo de células McCoy tratados con cicloheximida, técnica que tiene una sensibilidad de $70-85 \%$ en comparación con la $\mathrm{RPC}^{3}$. LW. Dicker y cols indican que parte del aumento en la prevalencia de infecciones por C. trachomatis detectada en el mundo se debería a una mayor sensibilidad de las técnicas de diagnóstico actualmente en uso $^{26}$.

Existe menos información con respecto a frecuencia de infección por $T$. vaginalis en mujeres gestantes en Chile. Un trabajo efectuado en Antofagasta, que incluyó a adolescentes embarazadas, detectó una frecuencia de infección de 5,7\%; otro realizado en la ciudad de Valdivia con gestantes de 12 a 43 años describe $T$. vaginalis en $7,5 \%$ de las pacientes ${ }^{24-25}$. Ambos estudios, como asimismo el nuestro, incluyeron el cultivo de $T$. vaginalis, técnica altamente sensible y específica, para el diagnóstico de este protozoo. Diferencias en los factores de riesgo de las poblaciones estudiadas explican claramente las diferencias en la frecuencia de infección.

En conclusión, aunque la frecuencia de C. trachomatis y de $T$. vaginalis, ha permanecido relativamente baja en el tiempo en la institución de estudio, por el creciente riesgo de resultado adverso perinatal asociado con estas ITS nos parece conveniente introducir la pesquisa rutinaria prenatal de estos microorganismos.

\section{Resumen}

Introducción: Las tasas epidemiológicas de gonorrea han disminuido constantemente en Chile, mientras que la incidencia de infecciones por Trichomonas vaginalis y Chlamydia trachomatis no es bien conocida. Estas infecciones de transmisión sexual (ITSs) están asociadas con resultados adversos del embarazo e infecciones perinatales. Objetivo: Actualizar su prevalencia en un hospital público de la Región Metropolitana de Chile. Pacientes y Métodos: Entre abril y octubre de 2010 y desde abril a octubre de 2011, fueron seleccionadas al azar mujeres embarazadas atendidas en el Servicio de atención prenatal del Hospital San Borja Arriarán para detección de $T$. vaginalis, $N$. gonorrhoeae y $C$. trachomatis, por cultivo en caldo Diamond modificado, cultivo en Thayer-Martin y mediante amplificación del gen omp1, por RPC anidada, respectivamente. Se excluyeron mujeres embarazadas que recibieron antimicrobianos los 30 días previos. Resultados: Se analizaron 255 muestras cérvico-vaginales. $C$. trachomatis fue detectada en 15 (5,9\%) de las mujeres embarazadas. T. vaginalis se aisló en $6(2,4 \%)$ de ellas, mientras que $N$. gonorrhoeae no se encontró en las gestantes. Conclusión: Los resultados muestran prevalencia baja de $C$. trachomatis y de $T$. vaginalis y ausencia de $N$. gonorrhoeae, proporción que se mantiene estable en este establecimiento desde la década de 1990s, con leve aumento de C. trachomatis.

\section{Referencias bibliográficas}

1.- World Health Organization, Departament of Reproductive Health and Research 2007. Global strategy for the prevention and control of sexually transmitted infections: 2006-2015: Breaking the chain of transmission. http://www. who.int/reproductivehealth/pl

2.- Mullick S, Watson-Jones D, Beksinska M, Mabey D. Sexually transmitted infections in pregnancy: prevalence, impact on pregnancy outcomes, and approach to treatment in developing countries. Sex Transm Infect 2005; 81: 294-302.

3.- Ovalle A, Martínez M A. Infección genital. Eghon Guzmán, Editor. Selección de temas de Gineco-Obstetricia Tomo II. 1 ed. Santiago de Chile: Publiimpacto; 2007, p. 875-923.

4.- Centers for Disease Control and Prevention. Sexually transmitted diseases treatment guidelines, 2010. MMWR Morb Mortal Wkly Rep 2010/59 (RR12): 1-114.
5.- Jani IV. The need for systematic evaluations of diagnostic tests. Clin Infect Dis 2010; 51: 609-10.

6.- Organización Mundial de la Salud (OMS). Global prevalence and incidence of selected curable sexually transmitted infections, overview and estimates. WHO/CDS/ EDC/2001.10.Geneva, 2001.

7.- Martínez M A, Reid I, Arias C, Napolitano C, Sandoval J, Molina R. Prevalencia de infección cervical por Chlamydia trachomatis en mujeres 
de la Región Metropolitana. Rev Med Chile 2008; 136: 1294-300.

8.- Huneeus A, Pumarino M G, Schilling A, Robledo P, Bofil M. Prevalencia de Chlamydia trachomatis y Neisseria gonorrhoeae en adolescentes chilenas. Rev Med Chile 2009; 137: 1569-74.

9.- Ovalle A, Martínez M A, Casals A, Yuhaniak R, Giglio M S. Estudio clínico y microbiológico de la enfermedad inflamatoria pélvica aguda. Rev Chil Obstet Ginecol 1993; 58:103-12.

10.- Blas M M, Canchihuaman F A, Alva I E, Hawes S E. Pregnancy outcomes in women infected with Chlamydia trachomatis: a population-based cohort study in Washington State. Sex Transm Infect 2007; 83: 314-8.

11.- Shaw J L, Dey S K, Critchley H O, Horne A W. Current knowledge of the aetiology of human tubal ectopic pregnancy. Hum Reprod Update 2010; 16: 432-44.

12.- Rours G I, Duijts L, Moll H A, Arends L R, de Groot R, Jaddoe V W, et al. Chlamydia trachomatis infection during pregnancy associated with preterm delivery: a populationbased prospective cohort study. Eur J Epidemiol 2011; 26: 493-502.

13.- Valencia C, Prado V, Ríos M, Cruz M A, Pilorget J J. Prevalencia de Chlamydia trachomatis en conjuntivitis neonatal determinada mediante las técnicas de inmunofluorescencia y amplificación génica.
Rev Med Chile 2000; 128: 758-65.

14.- Rosenman M B, Mahon B E, Downs S M, Kleiman M B. Oral erythromycin prophylaxis $v s$ watchful waiting in caring for newborns exposed to Chlamydia trachomatis. Arch Pediatr Adolesc Med 2003; 157: 565-71.

15.- Martínez MA, Millán F, González C. Chlamydia trachomatis genotypes associated with pneumonia in Chilean infants. Scand J Infect Dis 2009; 41: 313-6.

16.- Ovalle A, Martínez M A, Ferrand P, Peña V, Schwarze J E. Infección intraamniótica por Neisseria gonorrhoeae en un caso de rotura prematura de membranas de pretérmino. Rev Chil Obstet Ginecol 1999; 64: 73-8.

17.- Comité Normas Infecciones de Transmisión Sexual. Ministerio de Salud de Chile. Normas de manejo y tratamiento de infecciones de transmisión sexual (ITS). Primera parte. Rev Chilena Infectol 2009; 26: 174-90.

18.- Arancibia M C, Lemus Y. Frecuencia de colonización conjuntival por Chlamydia trachomatis y Neisseria gonorrhoeae en recién nacidos que recibieron quimioprofilaxis ocular con cloranfenicol. Tesis para optar al Título de Matrona. Universidad de Chile, Facultad de Medicina, Escuela de Obstetricia, 2010.

19.- Petrin D, Delgaty K, Bhatt R, Garber G. Clinical and microbiological aspects of Trichomonas vaginalis. Clin Microbiol Rev
1998; 11: 300-17.

20.- Cotch M F, Pastorek J G, Nugent R P, Hillier S L, Gibbs R S, Martin D H, et al. Trichomonas vaginalis associated with low birth weight and preterm delivery. Sex Transm Dis 1997; 24: 361-2.

21.- Carter J E, Whithaus K C. Neonatal respiratory tract involvement by Trichomonas vaginalis. A case report and review of the literature. Am J Trop Hyg 2008; 78: 17-9.

22.- Martínez M A, Barría A, Meneses R, Oyarzún P, Sandoval J. Vulvovaginitis en la adolescencia. Estudio etiológico. Rev Chil Obstet Ginecol 2003; 68: 499-502.

23.- Ovalle A, Martínez M A, Villagra E. Flora microbiana genital en embarazadas con y sin riesgo de infección. Rev Chil Obstet Ginecol 1996; 61: 5-11.

24.- Franjola T, Añazco R, Puente R, Moraleda L, Herrmann F, Palma M. Trichomonas vaginalis en embarazadas y en recién nacidos. Rev Med Chile 1989; 117: 142-5.

25.- Sagua H, Chiang V, Varela H, Neira I, Bugueño M, Contreras $\mathrm{C}$, et al. Trichomoniasis en adolescentes embarazadas de Antofagasta, Chile. Parasitología al Día 1999; 23: 9-14.

26.- Dicker L W, Mosure D J, Levine W C, Black C M, Berman S M. Impact of switching laboratory tests on reported trends in Chlamydia trachomatis infections. Am J Epidemiol 2000; 151: 430-5. 